\title{
Numerical Simulation for Bioconvection of Unsteady Stagnation Point Flow of Oldroyd-B Nanofluid with Activation Energy and Temperature-Based Thermal Conductivity Past a Stretching Disk
}

\author{
Muhammad Sami Rashad ${ }^{1}$, Haihu Liu ${ }^{1, *}$ and Shan Ali Khan ${ }^{2}$ \\ ${ }^{1}$ School of Energy and Power Engineering, Xian Jiaotong University, Xi'an, 710049, China \\ ${ }^{2}$ Department of Mathematics, Government College University Faisalabad, Layyah Campus, 31200, Pakistan \\ *Corresponding Author: Haihu Liu. Email: haihu.liu@xjtu.edu.cn
}

Received: 27 April 2021 Accepted: 22 June 2021

\begin{abstract}
A mathematical modeling is explored to scrutinize the unsteady stagnation point flow of Oldroyd-B nanofluid under the thermal conductivity and solutal diffusivity with bioconvection mechanism. Impacts of Joule heating and Arrhenius activation energy including convective boundary conditions are studied, and the specified surface temperature and constant temperature of wall (CTW) are discussed. The consequences of thermal conductivity and diffusivity are also taken into account. The flow is generated through stretchable disk geometry, and the behavior of non-linear thermal radiation is incorporated in energy equation. The partial differential equations governing the fluid flow in the structure is reduced into dimensionless nonlinear ODEs by applying suitable similarity variables. The obtained system of non-dimensional nonlinear ODEs is treated numerically with the help of bvp4c solver in Matlab under shooting algorithm. The impact of various prominent parameters on velocity profile, thermal profile, volumetric nanoparticle concentration and microorganism distribution is depicted in graphical form. The numerical outcomes for skin friction coefficient, heat transfer rate, Sherwood number as well as microorganism density number versus various parameters are listed in the tables. The results show that fluid velocity is reduced by increasing buoyancy ratio parameter, while the fluid flow increases with mixed convective parameter. The temperature profile is enhanced with the amount of nonlinear thermal radiation and temperature dependent thermal conductivity. Furthermore, concentration profiles of nanoparticles have opposite behavior for Brownian motion coefficient and thermophoresis diffusion parameter, and it is noticed that by varying Peclet number the microorganisms profile is declined. The proposed study is useful to control and optimize heat transfer in industrial applications.
\end{abstract}

\section{KEYWORDS}

Oldroyd-B nanofluid; thermal conductivity; solutal diffusivity conductivity; bioconvective microorganisms; shooting algorithm

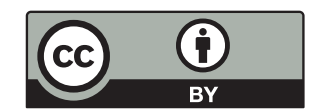

This work is licensed under a Creative Commons Attribution 4.0 International License, which permits unrestricted use, distribution, and reproduction in any medium, provided the original work is properly cited. 


\begin{tabular}{|c|c|}
\hline \multicolumn{2}{|c|}{ Nomenclature } \\
\hline PST & Surface temperature \\
\hline CWT & Constant wall temperature \\
\hline$\lambda_{1}$ & Relaxation time \\
\hline$p$ & Pressure of fluid \\
\hline$C$ & Concentration \\
\hline$N$ & Microorganisms \\
\hline$\alpha$ & Fluid heat diffusivity \\
\hline$D_{B}$ & Brownian diffusion coefficient \\
\hline$D_{T}$ & Brownian thermophoresis coefficient \\
\hline$k_{1}$ & Chemical reaction constant \\
\hline$h_{T}$ & Heat transfer coefficient \\
\hline$h_{C}$ & Mass transfer coefficient \\
\hline$S$ & Unsteadiness parameter \\
\hline$M$ & Magnetic parameter \\
\hline$N b$ & Brownian motion coefficient \\
\hline$\lambda$ & Mixed convection parameter \\
\hline$E c_{r}$ & Eckert number \\
\hline$N t$ & Thermophoresis diffusion parameter \\
\hline $\mathrm{Nr}$ & Buoyancy ratio parameter \\
\hline$N c$ & Bioconvection Rayleigh number \\
\hline$K_{1}$ & Chemical reaction parameter \\
\hline$E$ & Activation energy parameter \\
\hline$B i$ & Biot number \\
\hline$P e$ & Peclet number \\
\hline$\Omega$ & Microorganism difference variable \\
\hline$f^{\prime}$ & Characteristics of velocity distribution \\
\hline$\theta$ & Thermal profile \\
\hline$\phi$ & Solutal field of nanomaterials \\
\hline$\chi$ & Motile microorganism's profiles \\
\hline$E$ & Activation energy \\
\hline$P e$ & Peclet number \\
\hline $\operatorname{Pr}$ & Prandtl number \\
\hline$\theta_{w}$ & Temperature ratio parameter \\
\hline$R d$ & Radiation parameter \\
\hline Le & Lewis number \\
\hline$L b$ & Bioconvection Lewis number \\
\hline$S$ & Unsteadiness parameter \\
\hline$\alpha_{1}$ & Deborah number for relaxation to time parameter \\
\hline$\alpha_{2}$ & Deborah number for retardation to time parameter \\
\hline$\varepsilon_{1}$ & Thermal conductivity \\
\hline$\varepsilon_{2}$ & Solutal diffusivity parameter \\
\hline$K$ & Velocity ratio parameter \\
\hline$T$ & Temperature \\
\hline$C$ & Concentration \\
\hline
\end{tabular}

\section{Greek Symbols}

Density of fluid

$v \quad$ Kinematic viscosity 


\section{Introduction}

\subsection{Review of the Literature}

Transmission of heat integrated liquid flow is necessary for a significant number of nuclear and thermal-hydraulic systems. The involvement of liquids in the these systems can help to reduce capital costs, enhance operating efficiency and improve the performance of the system. Amongst various conventional methods, air is one of the main techniques of cooling electrical devices. The cooling is necessary in order to maintain the required thermal efficiency in different scientific and technological products such as computers, engines, chemical processes, and so on. Nanofluids, due to their exceptional thermo-physical characteristics and relatively slower heat resistance, have recently obtained the most attractive interest. Nanoparticles are comparatively tiny-sized particles that are contained in the fluid. The main purpose of introducing such particles are to enhance the heat conductivity of commonly utilized base liquids. The definition of nanofluid was initiated by Choi [1] who demonstrated that a host liquid, including water or ethylene-glycol fluid, can increase its heat efficiency by incorporating nanoparticles. Later, Buongiorno [2] identified Brownian motion and thermophoresis elements for the development of nanoparticles associated with heat transport. Aminian et al. [3] studied the impacts of $\mathrm{Al}_{2} \mathrm{O}_{3}-\mathrm{CuO}$-water-based nano liquid in a partition cylinder surrounded by a porous channel. Saha [4] evaluated the impact of the external electromagnetic field upon on free convection movement of the $\mathrm{Al}_{2} \mathrm{O}_{3}$-water nano liquid through the internal cylindrical wall. The radiation impacts under the Magnetohydrodynamic (MHD) movement of Casson-type nano liquid across a shrinking/stretched cylinder in the presence of chemical processes were reported by Bhandari et al. [5]. Mondal et al. [6] evaluated a steady 2-dimensional (MHD) Magnetohydrodynamic mixed convection flow of viscous nano liquid and heat transformation in an inclined stretched cylinder with a chemical process and a uniformly magnetic field. Basha et al. [7] discussed the properties of fluid transportation and entropy production of tangent hyperbolic nano liquid across a horizontal revolving cylinder with the impact of nonlinear Boussinesq estimation. Sheikholeslami et al. [8] studied the influence of the helical-twisting system on the thermal-hydraulic quantity of the nano liquid flow through tube. Sudarsana Reddy et al. [9] explored the thermal and solutal transformation properties of nano-liquid flow across a stretchable surface embedded in a porous medium. Mishra et al. [10] scrutinized the motion and thermal characteristics of magnetized silver-water $\left(\mathrm{Ag} / \mathrm{H}_{2} \mathrm{O}\right)$ nano liquid across a stretched cylinder. Zainal et al. [11] studied the occurrence of non-unique solutions in boundary layer flow owing to deformable surfaces under the implementation of specified surface heat flux. Saeed et al. [12] researched the heat properties of the Magnetohydrodynamic hybrid nano liquid $\left(\mathrm{Al}_{2} \mathrm{O}_{3}-\mathrm{Cu} / \mathrm{H}_{2} \mathrm{O}\right)$ flowing through permeable stretched cylinder in a Darcy medium. Jabbar et al. [13] observed the thermal properties of nanofluid in rotating cylinders under convective circumstances. Goudarzi et al. [14] evaluated the influence of nanomaterials migration in terms of Brownian motion coefficient and thermophoresis coefficient on natural convection of

$(A g-M g O) /$ hybrid-based nano liquid. The mathematical model of MHD mixed convective flow in a trapezoidal enclosure filled with a porous-saturated $\mathrm{Cu}$-water nano liquid was introduced by Ali et al. [15].

Mass exchange method coupled with chemical processes through activation energy is usually important in geothermal and oil reservoirs manufacturing, chemical engineering, and water and oil emulsion system, food preparation, and so on. It is important to establish conceptual outcomes for estimating the effect of activation energy across flows. The terminology Arrhenius activation energy was initially pioneered by Svante Arrhenius scientist in 1889. It is the lowest energy needed to transform the reactants into materials. Using a numerical method, Bestman [16] studied the free 
convection boundary layer flow when the boundary wall moves in its own plane via Arrhenius activation energy. Ramesh [17] studied viscoelastic nanofluid influenced by activation energy and chemical processes. Abuzaid et al. [18] investigated numerically the mixed convective movement of Oldroyd-B nano liquid subjected to Arrhenius activation energy and binary chemical processes. Waqas et al. [19] researched the nonlinear radiation flow of Eyring-Powell nano liquid with magnet dipoles and Arrhenius activation energy. The consequence of activation energy for the CarreauYasuda nanofluid flow was examined by Khan et al. [20].

Non-Newtonian liquids have become very unique in the manufacturing and engineering sectors. Commonly non-Newtonian fluids are divided into three categories: (I) the differential type fluids, (II) the rate type fluids, and (III) the integral type non-Newtonian fluids. Oldroyd-B fluid is an important sub-class of the rate-type fluid. Such fluids play an important role in industrial processes such as pharmaceutical industries, paper production, food processing, drug delivery, and cancer treatment. The Oldroyd-B fluid model was first introduced by Oldroyd in 1950 [21], motivated by the research of Fröhlich et al. [22] who developed a structured method for the improvement of viscoelastic fluid modeling techniques. The 2-dimensional flow of Oldroyd-B nano liquid through the nonlinearly stretchable sheet with the Cattaneo Christov heat flux model was studied by Ibrahim et al. [23]. The improvement of dual stratification in the nonlinear radiative movement of Oldroyd-B nano liquid was explained by Irfan et al. [24]. Anwar et al. [25] evaluated the consequence of Newtonian thermal conductivity by the unsteady natural convectional movement of Oldroyd-B nanofluid contained in an infinite, vertical stationary plate. Interested readers may refer to $[26,27]$ for more works on Oldroyd-B fluid.

Bioconvection is a naturally occurring phenomenon in swimming motile microorganisms. One type of motile microorganism, identified as the gyrotactic microorganism, is highly present in the aqueous system, which can swim in the flowing liquid along a specific direction with a fragile horizontal vortex and unstably fall in the flowing liquid with a significant horizontal vortex. Gyrotactic motile microorganism swimming components are important for understanding many ecological features of bioconvection. Microorganism molecules are used for the processing of agricultural materials including ethanol, fertilizers, biofuels, water plants as well as biodiesel. Bioconvection is an unrequested modification of macroscopic liquid patterns, including diminishing plumes. Two principal forms of up-swimming micro-organisms are normally utilized in convectional experimentation: low-heavy algae and solid oxytactic organisms. The terminology bioconvection was first proposed by Platt [28] in 1961, who described the formation of cells induced by microorganisms in the aqueous suspension. Mondal et al. [29] scrutinized the behavior of bioconvective phenomenon in nano liquid. Khan et al. [30] scrutinized the properties of a magnetic modified Newtonian fluid through bioconvection and chemically reactive organisms. Several investigators researched the bioconvection phenomenon, see [31-37]. Song et al. [38] conducted the analysis of the bioconvective 
phenomenon in micropolar nano liquid over the off-centered disk. The effects of exponential base heat source/sink and Cattaneo-Christov Laws on bioconvective Carreau nano liquid were analyzed by Farooq et al. [39]. Tlili et al. [40] scrutinized the use of Arrhenius energy with thermal radiative implications in the convectional flow of magnetic Oldroyd-B nanomaterials through a stretched cylinder. A mathematical method focusing on two-dimensional modified second-grade nano liquids against revolving Riga plate for the influence of the Arrhenius activation energy and bioconvection facets was examined by Waqas et al. [41].

In this work, a new class of fluid namely nanofluid is introduced, which has high thermal conductivity. Nanofluids are mostly utilized for their improved heat characteristics as a cooling medium in heat transformation equipment like radiators, cooling of microelectronics, and fuel cells. Inspired by the above studies, the main objective is to examine the impact of non-linearly thermal radiation on the stagnation point flow of bio-convective Oldroyd-B nano liquid past a stretchable disc with activation energy. The effects of temperature-based thermal efficiency and thermal diffusivity are investigated. The current model is explored for both constant wall temperature and prescribed surface temperature cases. The similarity transformations are used to obtain the ordinary differential equation from the developed system of PDEs. The numerical solution of ODEs is found by using the shooting scheme via the bvp4c MATLAB tool. The effect of flow governing parameters is illustrated for velocity profile, a thermal profile of species, concentration, and swimming microorganism field.

\section{Problem Modling}

Consider unsteady, 2-D stagnation point fluid flow of electrical conducting Oldroyd-B nano liquid with bioconvective swimming microorganism above a linearly stretching disk. Select a polar coordinate arrangement $(r, z)$ in which $r$-axes are taken in the horizontal directions over the stretched disk and $z$-axis is considered in the vertically upward direction respectively. The physical schematic and coordinate classification are exhibited in Fig. 1. The velocities are considered as $u_{w}(t, r)=\frac{a r}{1-\gamma t}$ and $u_{e}(t, r)=\frac{c r}{1-\gamma t}$, respectively, where $a, c \& \gamma$ are positive constants and the velocity $u_{w}(t, r)=\frac{a r}{1-\gamma t}$ is along the $z$ direction. The plane of the disk is exposed to the convective boundary conditions. In addition, the convective heat and mass transformation mechanisms are involved in the absence of heat production. With these assumptions, the physically governing boundary layer system of continuity, velocity, energy, the concentration of nanoparticles diffusion, and microorganism distribution in the occurrence of thermal radiative and magnetic fields over stretched disk can be described as [42,43].

$\frac{\partial(r u)}{\partial r}+\frac{\partial(r w)}{\partial z}=0$, 


$$
\begin{aligned}
& \frac{\partial u}{\partial t}+u \frac{\partial u}{\partial r}+w \frac{\partial u}{\partial z}+\lambda_{1}\left\{\frac{\partial^{2} u}{\partial t^{2}}+2 u \frac{\partial^{2} u}{\partial t \partial r}+2 w \frac{\partial^{2} u}{\partial t \partial z}+u^{2} \frac{\partial^{2} u}{\partial r^{2}}+2 u w \frac{\partial^{2} u}{\partial r \partial z}+w^{2} \frac{\partial^{2} u}{\partial z^{2}}\right\} \\
& =-\frac{1}{\rho} \frac{\partial p}{\partial r}+v\left[\frac{\partial^{2} u}{\partial z^{2}}\right]+\lambda_{2} v\left(\frac{\partial^{3} u}{\partial t \partial z^{2}}+w \frac{\partial^{3} u}{\partial z^{3}}+u \frac{\partial^{3} u}{\partial r \partial z^{2}}-2 \frac{\partial^{2} u}{\partial r \partial z} \frac{\partial u}{\partial z}-3 \frac{\partial u}{\partial r} \frac{\partial^{2} u}{\partial z^{2}}-\frac{\partial u}{\partial z} \frac{\partial^{2} w}{\partial z^{2}}\right) \\
& -\frac{\sigma \beta_{0}^{2}}{\rho}\left[u+\lambda_{1}\left\{\frac{\partial u}{\partial t}+w \frac{\partial u}{\partial z}\right\}\right]+\frac{1}{\rho_{f}}\left[\begin{array}{l}
\left(1-C_{f}\right) \rho_{f} \beta^{* *} g *\left(T-T_{\infty}\right) \\
-\left(\rho_{p}-\rho_{f}\right) g^{*}\left(C-C_{\infty}\right) \\
-\left(N-N_{\infty}\right) g^{*} \gamma\left(\rho_{m}-\rho_{f}\right)
\end{array}\right] \\
& \frac{\partial T}{\partial t}+u \frac{\partial T}{\partial r}+w \frac{\partial T}{\partial z}=\frac{1}{\rho c_{p}} \frac{\partial}{\partial z}\left[k(T) \frac{\partial T}{\partial z}\right]+\alpha\left[\frac{\partial^{2} T}{\partial z^{2}}\right]+\tau\left(D_{B} \frac{\partial T}{\partial z} \frac{\partial C}{\partial z}+\frac{D_{T}}{T_{\infty}}\left(\frac{\partial T}{\partial z}\right)^{2}\right) \\
& +Q_{0}\left(\frac{T-T_{\infty}}{\left(\rho c_{p}\right)_{f}}\right)+\frac{\sigma \beta_{0}^{2} u^{2}}{\left(\rho c_{p}\right)_{f}}+\frac{16 \sigma^{*}}{3 \rho c_{p} k^{*}} \frac{\partial}{z}\left(T^{3} \frac{\partial T}{\partial z}\right), \\
& \frac{\partial C}{\partial t}+u \frac{\partial C}{\partial r}+w \frac{\partial C}{\partial z}=\frac{1}{\rho c_{p}} \frac{\partial}{\partial z}\left[D(C) \frac{\partial C}{\partial z}\right]+D_{B}\left[\frac{\partial^{2} C}{\partial z^{2}}\right]+\frac{D_{T}}{T_{\infty}}\left[\frac{\partial^{2} T}{\partial z^{2}}\right] \\
& -k r^{2}\left(C-C_{\infty}\right)\left(\frac{T}{T_{\infty}}\right)^{n} \exp \left(\frac{-E_{a}}{K_{1} T}\right) \\
& \frac{\partial N}{\partial t}+u \frac{\partial N}{\partial r}+w \frac{\partial N}{\partial z}+\left[\frac{\partial}{\partial z}\left(N \frac{\partial C}{\partial z}\right)\right] \frac{b W_{c}}{\left(C_{w}-C_{\infty}\right)}=D_{m} \frac{\partial}{\partial z}\left(\frac{\partial N}{\partial z}\right),
\end{aligned}
$$

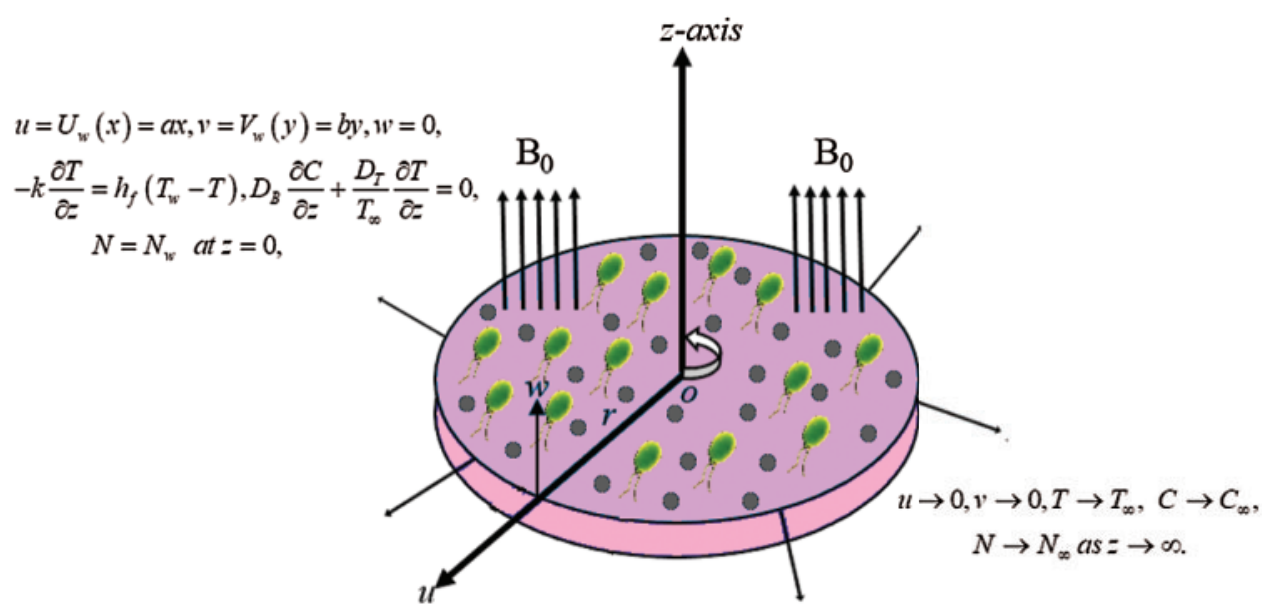

Figure 1: Physical layout of the flow 
with the appropriate constraints:

$$
\begin{aligned}
& u(t, r, z)=u_{w}(t, r)=\frac{a r}{1-\gamma t}, \quad w(t, r, z)=0, \\
& -k \frac{\partial T}{\partial z}=h_{T}\left(T_{w}-T\right), \quad D_{B} \frac{\partial C}{\partial z}+\frac{D_{T}}{T_{\infty}} \frac{\partial T}{\partial z}=0, \quad N=N_{w} \text { at } z=0, \\
& u=u_{e}=\frac{c r}{1-\gamma t}, \quad w \rightarrow 0, \quad T \rightarrow T_{\infty}, \quad C \rightarrow C_{\infty}, \quad N \rightarrow N_{\infty}, \text { as } z \rightarrow \infty .
\end{aligned}
$$

Here, the relaxation time is denoted by $\lambda_{1}, p, T, C$ and $N$ represent the fluid pressure, temperature, concentration, and bioconvection respectively; $v$ is the kinematic viscosity, $\alpha$ is the fluid heat diffusivity and $\rho$ is the fluid density, $D_{B}$ and $D_{T}$ are the Brownian motion and thermophoretic coefficients, $k_{1}$ is the chemical reaction constant, and $h_{T}$ and $h_{C}$ denote the heat and mass transfer coefficients.

Variable conductivities for heat and mass are given below:

$K(T)=k_{\infty}\left[1+\epsilon_{1}\left(\frac{T-T_{\infty}}{\Delta T}\right)\right], D(C)=D_{\infty}\left[1+\epsilon_{2}\left(\frac{C-C_{\infty}}{\Delta C}\right)\right]$.

With the similarity transformations given by $[44,45]$

$u=\frac{a r}{1-\gamma t} f^{\prime}(\zeta), \quad w=-2 \sqrt{\frac{a \nu}{1-\gamma t}} f(\zeta)$,

$T=T_{\infty}+\left(T_{w}-T_{\infty}\right) \theta(\zeta)(C W T)$,

$T=T_{\infty}+\frac{b r}{1-\gamma t} \theta(\zeta)(P S T), \quad \phi(\zeta)=\frac{C-C_{\infty}}{C_{w}-C_{\infty}}$,

$\chi(\zeta)=\frac{N-N_{\infty}}{N_{w}-N_{\infty}}, \quad \zeta=z \sqrt{\frac{a}{v(1-\gamma t)}}$,

Eqs. (2)-(5) reduce to the following forms:

$$
\begin{aligned}
& \frac{S}{2} \alpha_{2} \zeta f^{i v}-\alpha_{2} f f^{i v}-\alpha_{2} f^{\prime \prime 2}+2 \alpha_{2} S f^{\prime \prime \prime}-2 \alpha_{2} f^{\prime} f^{\prime \prime \prime}+f^{\prime \prime \prime}-\frac{S}{2} \zeta f^{\prime \prime}-S f^{\prime}-f^{\prime 2}+2 f f^{\prime \prime} \\
& \quad-\frac{7}{4} \alpha_{1} S^{2} \zeta f^{\prime \prime}-\frac{\alpha_{1}}{4} \zeta^{2} S^{2} f^{\prime \prime \prime}-2 \alpha_{1} S^{2} f^{\prime}-2 S \alpha_{1} f^{2}-\alpha_{1} \zeta S f^{\prime} f^{\prime \prime}+2 S \alpha_{1} \zeta f f^{\prime \prime \prime}+6 S \alpha_{1} f f^{\prime \prime} \\
& \quad+4 \alpha_{1} f f^{\prime} f^{\prime \prime}-4 \alpha_{1} f^{2} f^{\prime \prime \prime}-M\left(f^{\prime}+\alpha_{1} \frac{S}{2} \eta f^{\prime \prime}+\alpha_{1} S f^{\prime}-2 \alpha_{1} f f^{\prime \prime}-K-\alpha_{1} K S\right)+K S \\
& \quad+K^{2}+\alpha_{1}\left(2 K S^{2}+2 K^{2} S\right)+\lambda(\theta-N r \phi-N c \chi)=0
\end{aligned}
$$




$$
\begin{aligned}
& \left(1+\epsilon_{1} \theta\right) \theta^{\prime \prime}+\epsilon_{1} \theta^{\prime 2}+\frac{4}{3} R d\left[\begin{array}{l}
\theta^{\prime \prime}+\left(\theta_{w}-1\right)^{3}\left(3 \theta^{2} \theta^{\prime 2}+\theta^{3} \theta^{\prime \prime}\right) \\
+3\left(\theta_{w}-1\right)^{2}\left(2 \theta \theta^{\prime 2}+\theta^{2} \theta^{\prime \prime}\right) \\
+3\left(\theta_{w}-1\right)\left(\theta^{\prime 2}+\theta \theta^{\prime \prime}\right)
\end{array}\right]+\operatorname{Pr}\left(2 f \theta^{\prime}-\frac{S}{2} \zeta \theta^{\prime}\right) \\
& +\operatorname{Pr} N b \theta^{\prime} \phi^{\prime}+\operatorname{Pr} N t \theta^{\prime 2}+\operatorname{PrMEc}_{r} f^{\prime 2}+\operatorname{Pr} \delta \theta=0, \quad(C W T) \\
& \left(1+\epsilon_{1} \theta\right) \theta^{\prime \prime}+\epsilon_{1} \theta^{\prime 2}+\frac{4}{3} R d\left[\begin{array}{l}
\theta^{\prime \prime}+\left(\theta_{w}-1\right)^{3}\left(3 \theta^{2} \theta^{\prime 2}+\theta^{3} \theta^{\prime \prime}\right) \\
+3\left(\theta_{w}-1\right)^{2}\left(2 \theta \theta^{\prime 2}+\theta^{2} \theta^{\prime \prime}\right) \\
+3\left(\theta_{w}-1\right)\left(\theta^{\prime 2}+\theta \theta^{\prime \prime}\right)
\end{array}\right]+\operatorname{Pr}\left(2 f \theta^{\prime}-S \theta-\theta f^{\prime}-\frac{S}{2} \zeta \theta^{\prime}\right) \\
& +\operatorname{Pr} N b \theta^{\prime} \phi^{\prime}+\operatorname{Pr} N t \theta^{\prime 2}+\operatorname{PrMEc}_{r} f^{\prime 2}+\operatorname{Pr} \delta \theta=0, \quad(P S T) \\
& \left(1+\epsilon_{2}\right) \phi^{\prime \prime}+\epsilon_{2} \phi^{\prime 2}+L e \operatorname{Pr}\left(2 f \phi^{\prime}-\frac{S}{2} \zeta \phi^{\prime}\right)+L e \operatorname{Pr} \frac{N t}{N b} \theta^{\prime \prime} \\
& -L e \operatorname{Pr} \sigma(1+\delta \theta)^{n} \exp \left(\frac{-E}{1+\delta \theta}\right) \phi=0, \\
& \chi^{\prime \prime}+L b\left(2 f \chi^{\prime}-\frac{S}{2} \zeta \chi^{\prime}\right)-P e\left[\chi^{\prime} \phi^{\prime}+(\Omega+\chi) \phi^{\prime \prime}\right]=0,
\end{aligned}
$$

with

$f(0)=0, \quad f^{\prime}(0)=1, \quad \theta^{\prime}(0)=-B i(1-\theta(0))$,

$\phi^{\prime}(0)+\frac{N t}{N b} \theta^{\prime}(0)=0, \quad \chi(0)=1$,

$f^{\prime}(\infty)=K, \quad \theta(\infty)=0, \quad \phi(\infty)=0, \quad \chi(\infty)=0$.

In the above equations, $S=\frac{\gamma}{a}$ represents the unsteadiness parameter, $\alpha_{1}=\frac{\lambda_{1} a}{1-\gamma t}$ is the Deborah number of relaxation time, $\alpha_{2}=\frac{a \lambda_{2}}{1-\gamma t}$ is the Deborah number of retardation time. $K=\frac{c}{a}$ is the velocity ratio parameter, $M=\frac{\sigma B_{0}^{2}(1-\gamma t)}{\left(\rho_{f}\right) a}$ denotes the magnetic parameter, $N b=$ $\frac{\tau D_{B}(\Delta C)}{v}$ is the Brownian motion parameter, $E c_{r}=\frac{u_{w}^{2}}{c_{p} \Delta T(1-\gamma t)}$ is the Eckert number, $\lambda=$ $\frac{\beta^{* *} g *\left(1-C_{\infty}\right)\left(T_{w}-T_{\infty}\right)}{a(1-\gamma t)}$ represents the Mixed convection parameter, $N t=\frac{\tau D_{T}(\Delta T)}{\nu T_{\infty}}$ stands for thermophoresis parameter, $N r=\frac{\left(\rho_{p}-\rho_{f}\right)\left(C_{w}-C_{\infty}\right)}{\rho_{f}\left(1-C_{\infty}\right)\left(T_{w}-T_{\infty}\right) \beta^{* *}}$ is the Buoyancy ratio parameter, and $N c=\frac{\gamma *\left(\rho_{m}-\rho_{f}\right)\left(N_{w}-N_{\infty}\right)}{\rho_{f}\left(1-C_{\infty}\right)\left(T_{w}-T_{\infty}\right) \beta^{* *}}$ is the Bioconvectional Rayleigh number. Prandtl number 
is symbolized by $\operatorname{Pr}=\frac{v}{\alpha_{1}}, \delta=\frac{Q_{0}(1-\gamma t)}{a\left(\rho c_{p}\right)}$ is the heat source/sink parameter, $K_{1}=\frac{k_{1}(1-\gamma t)}{a}$ represents the chemical reaction, $E=\frac{E_{a}}{K_{1} T_{\infty}}$ stands for the activation energy, $L e=\frac{\alpha_{1}}{D_{B}}$ is the Lewis number, $B i=\frac{h_{T}}{k} \sqrt{\frac{v(1-\gamma t)}{a}}$ is the Biot number, the bioconvection Lewis number is symbolized by $L b=\frac{v}{D_{m}}$, the Peclet number is denoted as $P e=\frac{b W_{c}}{D_{m}}$, and the microorganisms difference variable is represented by $\Omega=\frac{N_{\infty}}{N_{w}-N_{\infty}}$.

The engineering quantities of interest include:

$C_{f}=\frac{\sqrt{\tau_{z r}^{2}+\tau_{z \varphi}^{2}}}{\rho(a r)^{2}}, \quad N u_{r}=\left.\frac{r}{\left(T_{w}-T_{\infty}\right)}\left(1+\frac{16 \sigma^{*} T^{3}}{3 \rho c_{p} k^{*}}\right)\left(\frac{\partial T}{\partial z}\right)\right|_{z=0}$
$S h_{r}=-\left.\frac{r}{\left(C_{w}-C_{\infty}\right)}\left(\frac{\partial C}{\partial z}\right)\right|_{z=0}, \quad S n_{r}=-\left.\frac{r}{\left(N_{w}-N_{\infty}\right)}\left(\frac{\partial N}{\partial z}\right)\right|_{z=0}$

By adopting similarity transformations, the dimensionless form of engineering quantities are:

$C_{f} \operatorname{Re}_{r}^{0.5}=\sqrt{f^{\prime \prime 2}(0)}$,

$R e^{-0.5} N u_{r}=-\left(1+\frac{4}{3}\left(1+\left(\theta_{w}-1\right) \theta(0)\right)^{3}\right) \theta^{\prime}(0)$,

$\operatorname{Re}^{-0.5} S h_{r}=-\phi^{\prime}(0)$,

$\operatorname{Re}^{-0.5} S n_{r}=-\chi^{\prime}(0)$.

\section{Numerical Algorithm}

In this section, the obtained dimensionless nonlinear (ODEs) (9)-(13) subject to the boundary constraints (14), (15) are numerically integrated by applying bvp4c solver in MATLAB software. The shooting method is utilized to transform the higher-order non-dimensional flow system into the first ordered boundary value problem. For this purpose, we introduce some new variables such as:

Let us suppose that the transformed variables as

$$
\begin{aligned}
& f=y_{1}, \quad f_{\zeta}=y_{2}, \quad f_{\zeta \zeta}=y_{3}, \quad f_{\zeta \zeta \zeta}=y_{4}, \quad f_{\zeta \zeta \zeta \zeta}=y_{4}^{\prime} \\
& \theta=y_{5}, \quad \theta_{\zeta}=y_{6}, \quad \theta_{\zeta \zeta}=y_{6}^{\prime} \\
& \phi=y_{7}, \quad \phi_{\zeta}=y_{8}, \quad \phi_{\zeta \zeta}=y_{8}^{\prime}, \\
& \chi=y_{9}, \quad \chi_{\zeta}=y_{10}, \quad \chi_{\zeta \zeta}=y_{10}^{\prime},
\end{aligned}
$$




$$
\begin{aligned}
& y_{4}^{\prime}=\frac{\left[\begin{array}{l}
-y_{4}-2 S \alpha_{1} \zeta y_{1} y_{4}+\frac{\alpha_{1}}{4} \zeta^{2} S^{2} y_{4}+4 \alpha_{1} y_{1}^{2} y_{4} \frac{S}{2} \zeta y_{3}+S y_{2}+y_{2}^{2}-2 y_{1} y_{3} \\
+\frac{7}{4} \alpha_{1} S^{2} \zeta y_{3}+2 \alpha_{1} S^{2} y_{2}+2 S \alpha_{1} y_{2}^{2}+\alpha_{1} \zeta S y_{2} y_{3}-6 S \alpha_{1} y_{1} y_{3}-4 \alpha_{1} y_{1} y_{2} y_{3} \\
+M\left(y_{2}+\alpha_{1} \frac{S}{2} \zeta y_{3}+\alpha_{1} S y_{2}-2 \alpha_{1} y_{1} y_{3}-K-\alpha_{1} K S\right)-K S-K^{2}-\alpha_{1}\left(\begin{array}{l}
2 K S^{2} \\
+2 K^{2} S
\end{array}\right) \\
-\alpha_{2} y_{3}^{2}-2 \alpha_{2} S y_{4}+2 \alpha_{2} y_{2} y_{4} y_{2} y_{3}-\lambda\left(y_{4}-N r y_{6}-N c y_{8}\right)
\end{array}\right]}{\left[\frac{S}{2} \alpha_{2} \zeta-\alpha_{2} y_{1}\right]}, \\
& -\epsilon_{1} y_{6}^{2}-\frac{4}{3} R d\left[\left(\theta_{w}-1\right)^{3}\left(3 y_{5}^{2} y_{6}^{2}\right)+3\left(\theta_{w}-1\right)^{2}\left(2 y_{5} y_{6}^{2}\right)+3\left(\theta_{w}-1\right) y_{6}^{2}\right] \\
& y_{6}^{\prime}=\frac{-\operatorname{Pr}\left(2 y_{1} y_{6}-\frac{S}{2} \zeta y_{6}\right)-\operatorname{Pr} N b y_{6} y_{8}-\operatorname{Pr} N t y_{6}^{2}-\operatorname{PrMEc} y_{2} y_{2}^{2} \operatorname{Pr} \delta y_{4}}{\left.\left(1+\epsilon_{1}\right)+\frac{4}{3} R d\left(1+\left(\theta_{w}-1\right)^{3} y_{5}^{3}\right\}+3\left(\theta_{w}-1\right)^{2} y_{5}^{2}+3\left(\theta_{w}-1\right) y_{5}\right)},(C W T) \\
& -\epsilon_{1} y_{6}^{2}-\frac{4}{3} R d\left[\left(\theta_{w}-1\right)^{3}\left(3 y_{5}^{2} y_{6}^{2}\right)+3\left(\theta_{w}-1\right)^{2}\left(2 y_{5} y_{6}^{2}\right)+3\left(\theta_{w}-1\right) y_{6}^{2}\right] \\
& -\operatorname{Pr}\left(2 y_{1} y_{6}-S y_{5}-y_{5} y_{2}-\frac{S}{2} \zeta y_{6}\right)-\operatorname{Pr} N b y_{6} y_{8}-\operatorname{Pr} N t y_{6}^{2}-\operatorname{PrMEc}_{r} y_{2}^{2} \\
& y_{6}^{\prime}=\frac{-\operatorname{Pr} \delta y_{5}}{\left.\left(1+\epsilon_{1}\right)+\frac{4}{3} R d\left(1+\left(\theta_{w}-1\right)^{3} y_{5}^{3}\right\}+3\left(\theta_{w}-1\right)^{2} y_{5}^{2}+3\left(\theta_{w}-1\right) y_{5}\right)},(P S T) \\
& -\epsilon_{2} y_{8}^{2}-L e \operatorname{Pr}\left(2 y_{1} y_{8}-\frac{S}{2} \zeta y_{8}\right)-L e \operatorname{Pr} \frac{N t}{N b} y_{6}^{\prime} \\
& y_{8}^{\prime}=\frac{+L e \operatorname{Pr} \sigma\left(1+\delta y_{5}\right)^{n} \exp \left(\frac{-E}{1+\delta y_{5}}\right) y_{7}}{\left(1+\epsilon_{2}\right)} \text {, } \\
& y_{10}^{\prime}=-L b\left(2 y_{1} y_{10}-\frac{S}{2} \zeta y_{10}\right)+P e\left[y_{10} y_{8}+\left(\Omega+y_{9}\right) y_{8}^{\prime}\right] \text {, } \\
& y_{1}(0)=0, \quad y_{2}(0)=1, \quad y_{6}(0)=-B i\left(1-y_{5}(0)\right), \\
& y_{8}(0)+\frac{N t}{N b} y_{6}(0)=0, \quad y_{9}(0)=1 \text {, } \\
& y_{2}(\infty)=K, \quad y_{5}(\infty)=0, \quad y_{7}(\infty)=0, \quad y_{9}(\infty)=0 .
\end{aligned}
$$

\section{Result and Discussion}

The unsteady natural convection of Oldroyd-B nano liquid via stretchable disk has been investigated. The numerical solution is obtained by utilizing MATLAB inbuilt function bvp4c, and the physical characteristics of the nanofluid is described by Buongiorno model. This section scrutinizes the influence of important physical parameters on quantities of substantial concentration. As sketched in Fig. 1, our aim is to visualize the characteristics of velocity distribution $f^{\prime}$, thermal profiles $\theta$, solutal field of nanomaterials $\phi$, and motile microorganism profiles $\chi$ against an involved physical parameter such as the Brownian diffusion coefficient $\mathrm{Nb}$, the thermophoretic diffusion coefficient $N t$, the activation energy $E$, the Peclet number $P e$, the Mixed convection parameter $\lambda$, the Prandtl number Pr, the temperature ratio parameter $\theta_{w}$, the Radiation parameter $R d$, the Lewis number $L e$, the velocity ratio parameter $K$, the Lewis number $L b$, the magnetic 
parameter $M$, the Buoyancy ratio parameter $N r$, the Bioconvectional Rayleigh number $N c$, the unsteadiness parameter $S$, the Deborah number of relaxation time $\alpha_{1}$, the Deborah number of retardation time $\alpha_{2}$, the thermal conductivity variable $\varepsilon_{1}$, the solutal diffusivity parameter $\varepsilon_{2}$. These plots are all shown Figs. 2-15.

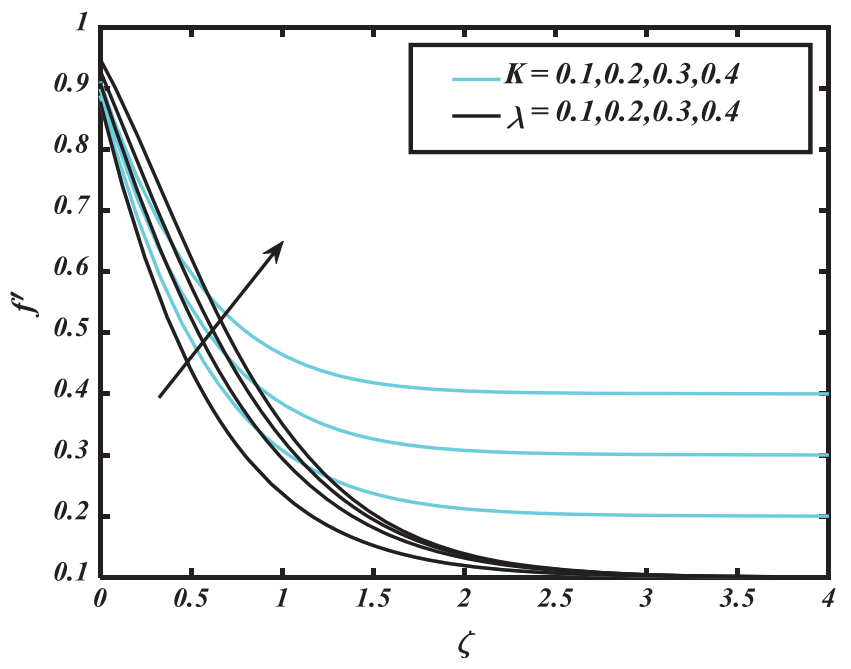

Figure 2: $f^{\prime}$ vs. $K \& \lambda$

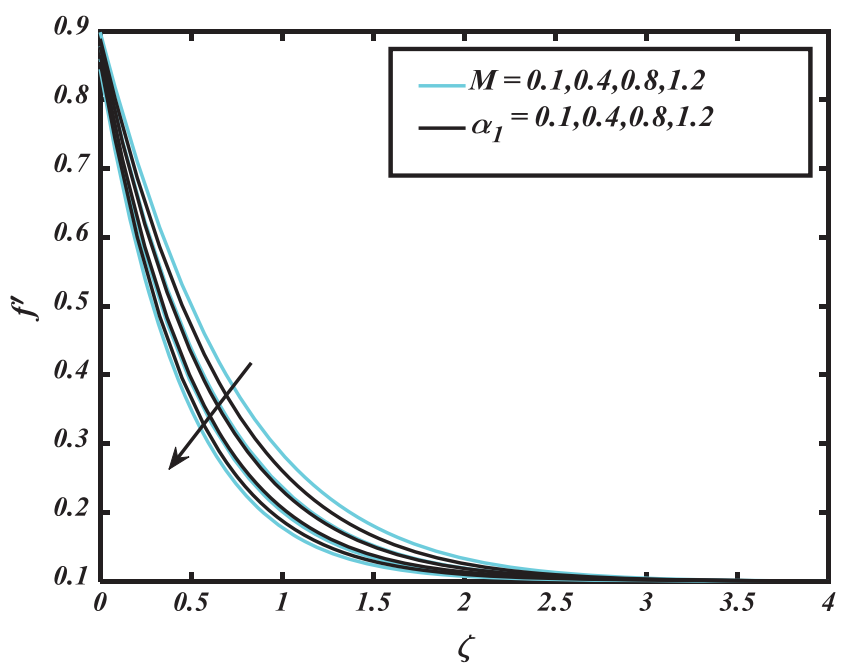

Figure 3: $f^{\prime}$ vs. $M \& \alpha_{1}$

Fig. 2 explores the effect of mixed convective parameter $\lambda$ and velocity ratio parameter $K v s$. the flow of fluid $f^{\prime}$. The fluid velocity is raised by enhancing the mixed convection parameter $\lambda$. Further from these curves lines it is noticed that the flow of fluid $f^{\prime}$ also improves for greater velocity ratio parameter $K$. Fig. 3 is confined to discuss the inspiration of magnetic parameter $M$ and Deborah number of relaxation time $\alpha_{1} v s$. velocity profile $f^{\prime}$. The larger magnet parameter $M$ and Deborah number of relaxation to time parameter $\alpha_{1}$ diminish the velocity profile. Practically, 
a larger magnetic parameter produces the Lorenz forces which cause the diminution in the flow of fluid. Fig. 4 provides the information about the inspiration of the buoyancy ratio parameter $\mathrm{Nr}$ and Deborah number of retardation time $\alpha_{2}$ across velocity field $f^{\prime}$. Velocity field $f^{\prime}$ declines for the highest estimation buoyancy ratio parameter $N r$, while it shows opposite behavior for $\alpha_{2}$. Features of unsteady parameter $S$ and bioconvection Rayleigh number $N c$ vs. the flow of fluid $f^{\prime}$ are illustrated in Fig. 5. It is clear that the momentum distribution $f^{\prime}$ declines with enlarging the variation of unsteadiness parameter $S$ and bioconvection Rayleigh number $N c$.

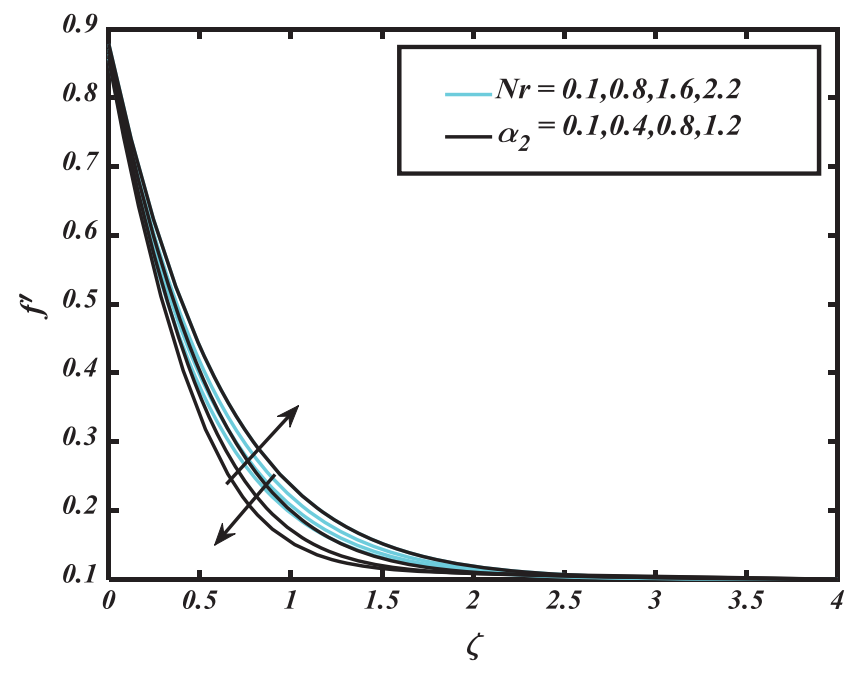

Figure 4: $f^{\prime}$ vs. $N r \& \alpha_{2}$

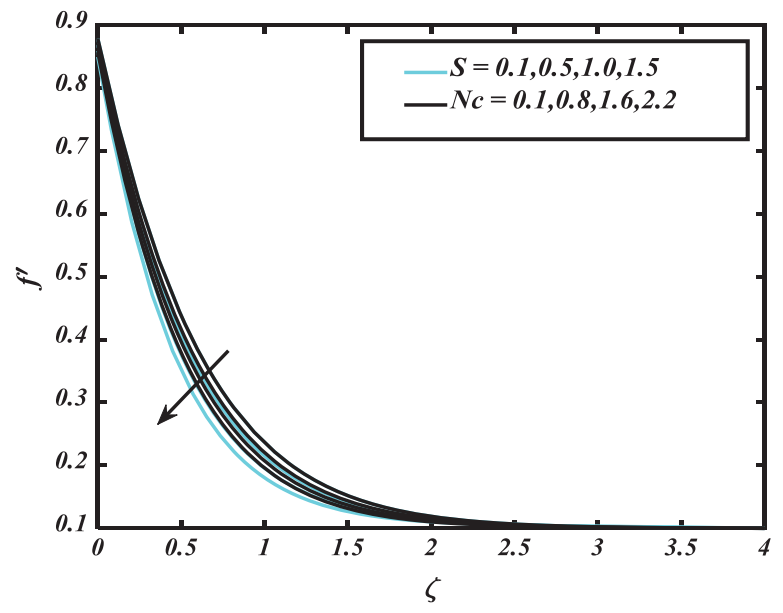

Figure 5: $f^{\prime}$ vs. $S \& N c$

The influence of radiation parameter $R d$ on a thermal profile $\theta$ in the case of constant wall temperature and prescribed surface temperature is shown in Fig. 6. From this figure, it is seen that temperature distribution $\theta$ exaggerates with radiation parameter $R d$. Fig. 7 is presented to check the effect of the temperature ratio parameter $\theta_{w}$ on the temperature profile $\theta$. It is witnessed that 
the temperature profile $\theta$ boosts up for greater temperature ratio parameter $\theta_{w}$. Fig. 8 is plotted to show the temperature distribution $\theta$ vs. the variable thermal conductivity $\epsilon_{1}$ for both constant wall temperature and prescribed surface temperature. Increasing variable thermal conductivity $\epsilon_{1}$ leads to higher temperature distribution $\theta$. In Fig. 9, the effect of Prandtl number $\operatorname{Pr}$ on the thermal field of species $\theta$ for both constant wall temperature and prescribed surface temperature is illustrated. Thermal field $\theta$ reduces with the rise in Prandtl number Pr. Fig. 10 describes the temperature profile $\theta$ for different values of the thermophoretic parameter $N t$. It is seen that the thermal field $\theta$ increases with enlarging the thermophoresis parameter $N t$ for both constant wall temperature and prescribed surface temperature. Physically, the thermophoresis forces are developed due to increment in thermophoretic parameter which causes the movement of the tiny-sized nanoparticles from a warm surface to the cold region and thus the enhancement of the temperature field.

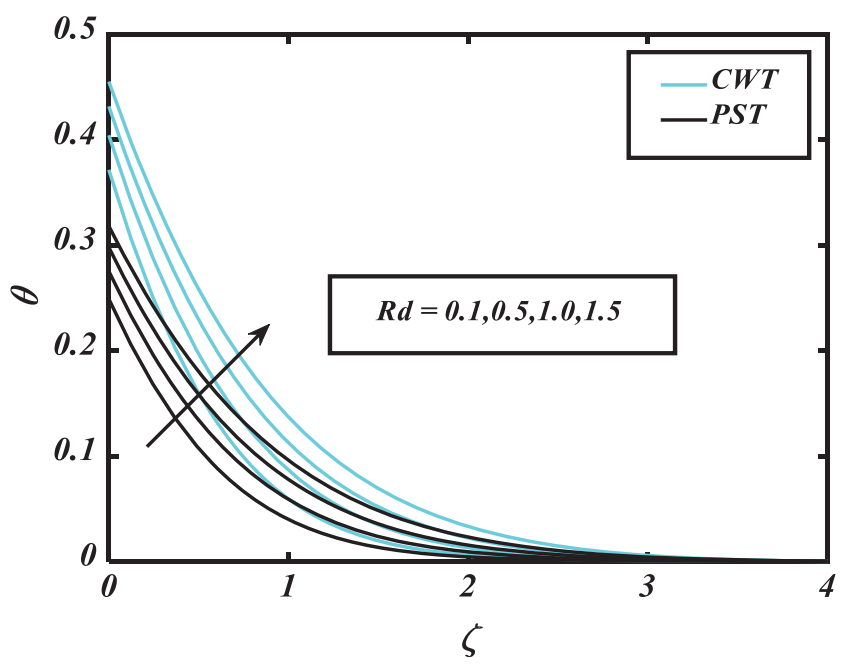

Figure 6: $\theta$ vs. $R d$

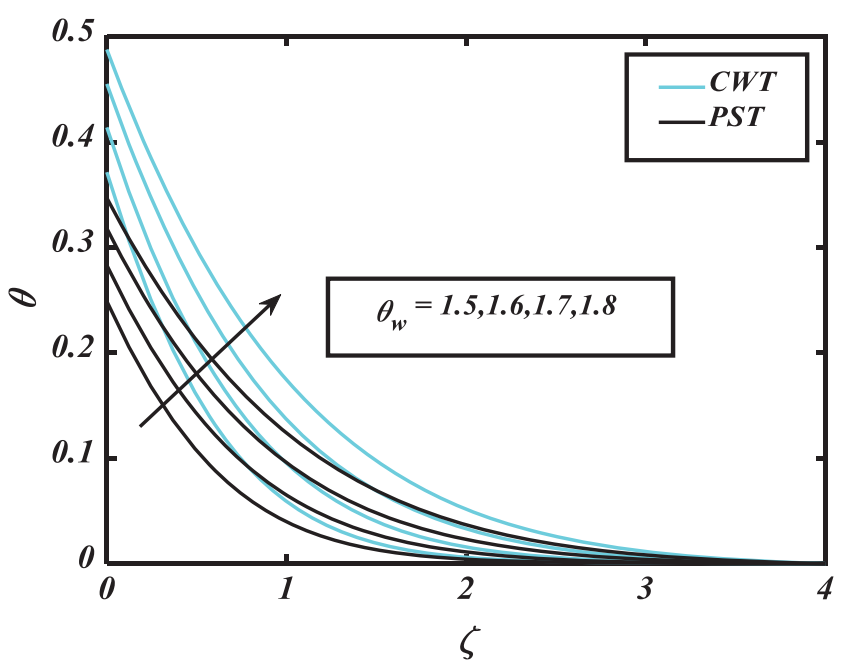

Figure 7: $\theta$ vs. $\theta_{w}$ 


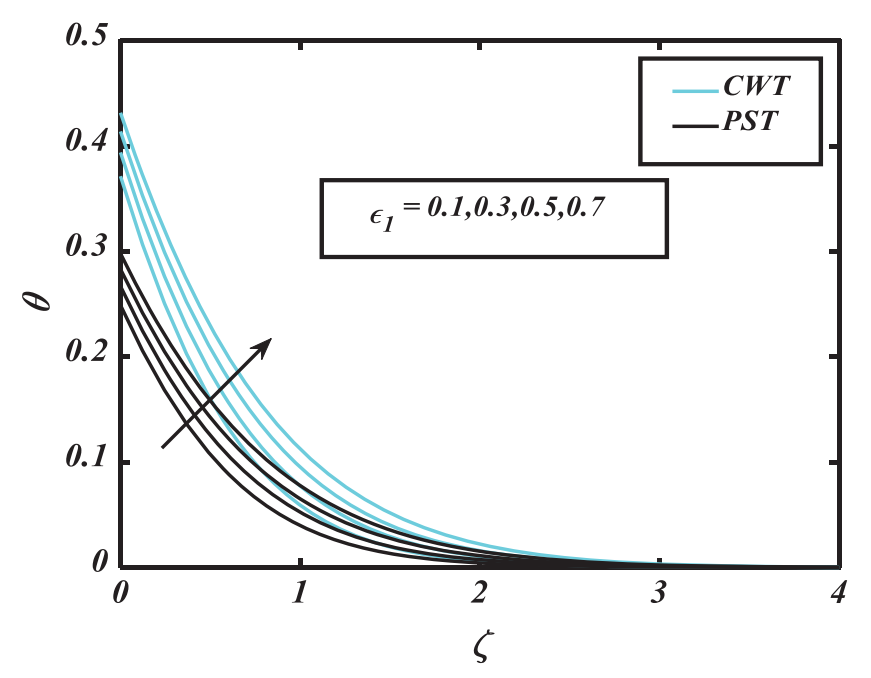

Figure 8: $\theta$ vs. $\quad \varepsilon_{1}$

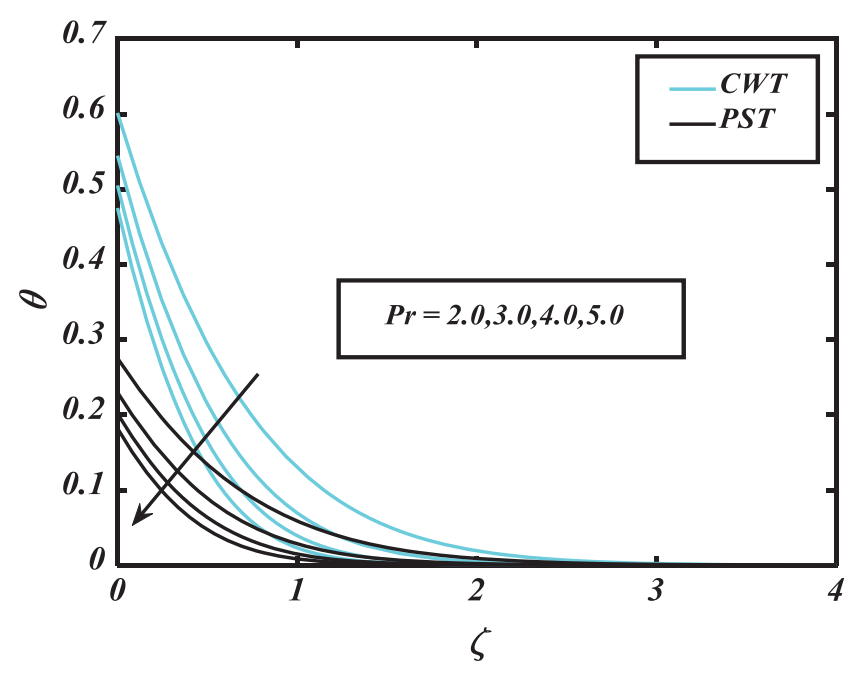

Figure 9: $\theta$ vs. $\operatorname{Pr}$

Fig. 11 shows how the mixed convection parameter $\lambda$ and Brownian motion coefficient $N b$ influence the solutal field of species $\phi$. Clearly, increasing mixed convection parameter and Brownian diffusion coefficient diminish the concentration $\phi$. Fig. 12 is plotted to elucidate the significance of the Prandtl number and Lewis number against the concentration of species $\phi$. It is found that an increment in the Prandtl number $\operatorname{Pr}$ or Lewis number Le depreciates the nanoparticle concentration field $\phi[46,47]$. To scrutinize the consequence of velocity ratio parameter $K$ and thermophoresis parameter $N t v s$. volumetric concentration of nanoparticles $\phi$, Fig. 13 is designed. It is shown that nanoparticle concentration field $\phi$ is the rising function of velocity ratio parameter $K$ and thermophoresis parameter $N t$. From Fig. 14 it is noticed that $\phi$ is an uplifting function of solutal diffusivity $\epsilon_{1}$ and Arrhenius activation energy parameter $E$. The significance of the Peclet number $P e$ and the bioconvection Lewis number $L b$ against swimming microorganism field $\chi$ is depicted in Fig. 15. We can see that the microorganism field $\chi$ reduces for greater Peclet number $P e$ or bioconvection Lewis number $L b$. 


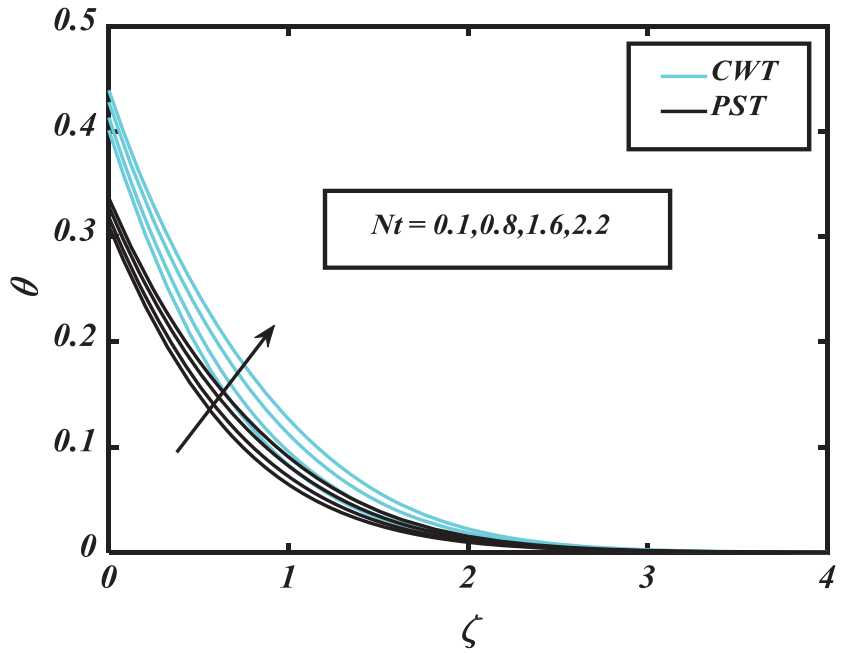

Figure 10: $\theta$ vs. $N t$

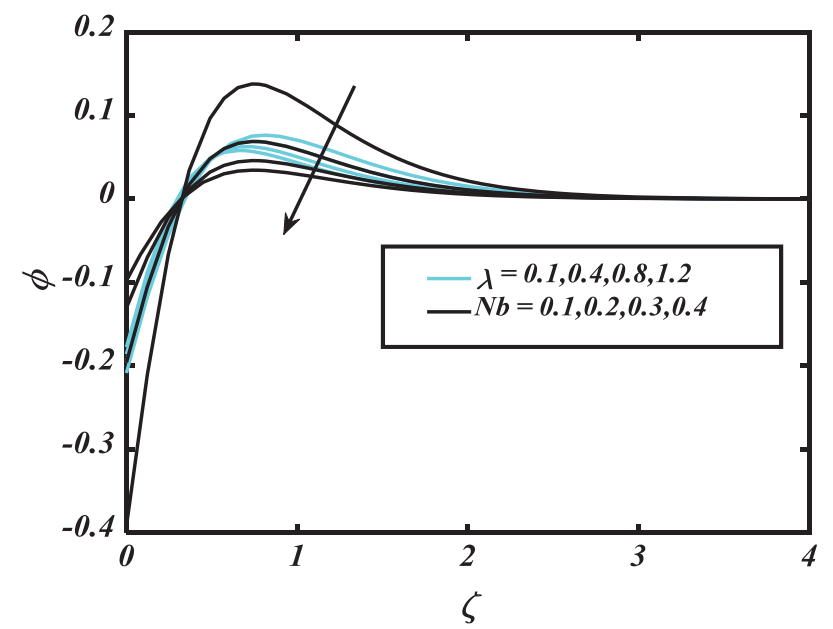

Figure 11: $\phi$ vs. $\lambda \& N b$

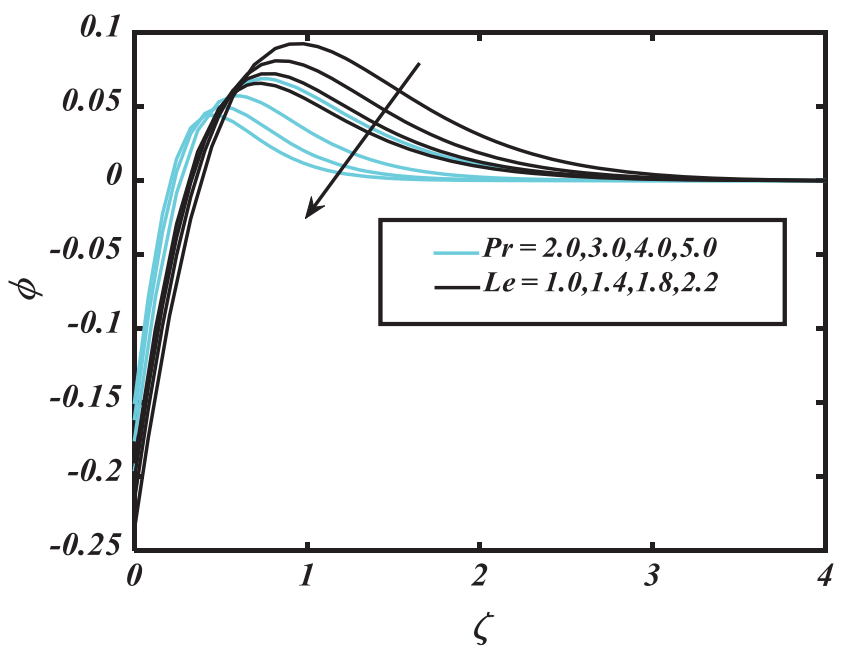

Figure 12: $\phi$ vs. $\operatorname{Pr} \& L e$ 


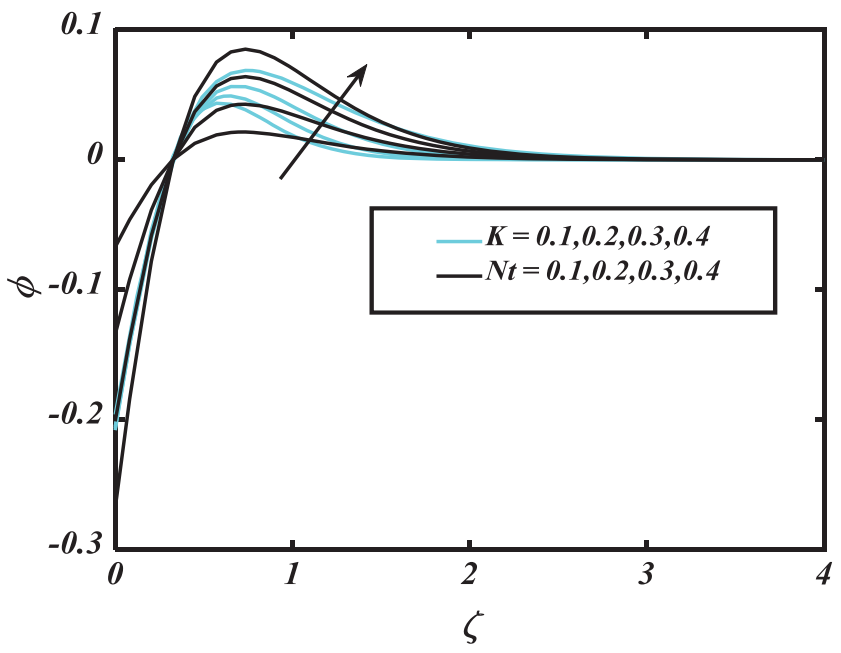

Figure 13: $\phi$ vs. $K \& N t$

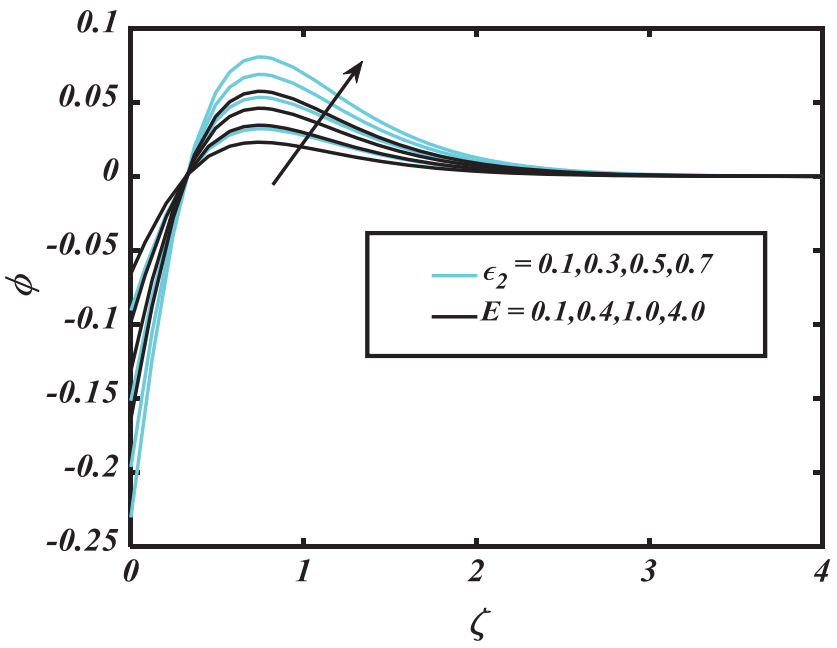

Figure 14: $\phi$ vs. $\varepsilon_{2} \& E$

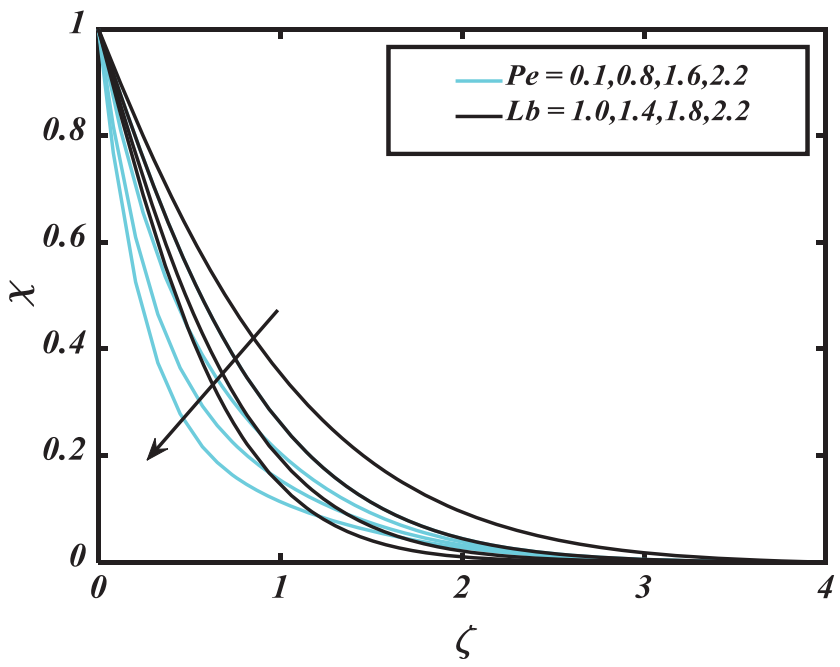

Figure 15: $\chi$ vs. $P e \& L b$ 
Table 1 displays that drag forces coefficient $-f^{\prime \prime}(0)$ increases with $N r$ and $N c$, but decreases with $\lambda$. Tables 2 and 3 depict that heat rate $-\theta^{\prime}(0)$ and local Sherwood number $-\phi^{\prime}(0)$ are reduced with growing $\theta_{w}$ but rises with $\operatorname{Pr}$. Table 4 displays that the local density number of swimming microorganisms increases with $P e$ and $L b$.

Table 1: Estimations of local skin friction coefficient $-f^{\prime \prime}(0)$ for different parameters

\begin{tabular}{|c|c|c|c|c|c|c|}
\hline$M$ & $\lambda$ & $N r$ & $N c$ & $K$ & $S$ & $-f^{\prime \prime}(0)$ \\
\hline 0.1 & 0.1 & 0.5 & 0.5 & 0.3 & 0.2 & 1.0101 \\
\hline 0.6 & & & & & & 1.1280 \\
\hline 1.2 & & & & & & 1.2517 \\
\hline \multirow[t]{3}{*}{0.5} & 0.2 & 0.5 & 0.5 & 0.3 & 0.2 & 1.0914 \\
\hline & 0.8 & & & & & 1.0087 \\
\hline & 1.6 & & & & & 0.9042 \\
\hline \multirow[t]{3}{*}{0.5} & 0.1 & 0.2 & 0.5 & 0.3 & 0.2 & 1.09734 \\
\hline & & 0.8 & & & & 1.1324 \\
\hline & & 1.6 & & & & 1.2456 \\
\hline \multirow[t]{3}{*}{0.5} & 0.1 & 0.5 & 0.2 & 0.3 & 0.2 & 1.1022 \\
\hline & & & 0.8 & & & 1.1723 \\
\hline & & & 1.6 & & & 1.2768 \\
\hline \multirow[t]{3}{*}{0.5} & 0.1 & 0.5 & 0.5 & 0.2 & 0.2 & 1.0980 \\
\hline & & & & 0.6 & & 1.00812 \\
\hline & & & & 1.2 & & 0.8912 \\
\hline \multirow[t]{3}{*}{0.5} & 0.1 & 0.5 & 0.5 & 0.3 & 0.1 & 1.0101 \\
\hline & & & & & 0.5 & 1.1367 \\
\hline & & & & & 1.0 & 0.2648 \\
\hline
\end{tabular}

Table 2: Outcomes of local Nusselt number $-\theta^{\prime}(0)$ for various parameters

\begin{tabular}{|c|c|c|c|c|c|c|}
\hline$M$ & $\lambda$ & $P r$ & $N t$ & $B i$ & $\theta_{w}$ & $-\theta^{\prime}(0)$ \\
\hline 0.1 & 0.1 & 2.0 & 0.3 & 2.0 & 0.4 & 0.8276 \\
\hline 0.6 & & & & & & 0.8099 \\
\hline 1.2 & & & & & & 0.7982 \\
\hline \multirow[t]{3}{*}{0.5} & 0.2 & & 0.3 & 2.0 & 0.4 & 0.8147 \\
\hline & 0.8 & & & & & 0.8228 \\
\hline & 1.6 & & & & & 0.8323 \\
\hline \multirow[t]{3}{*}{0.5} & 0.1 & 3.0 & 0.3 & 2.0 & 0.4 & 0.9107 \\
\hline & & 4.0 & & & & 0.9900 \\
\hline & & 5.0 & & & & 1.0500 \\
\hline \multirow[t]{3}{*}{0.5} & 0.1 & 2.0 & 0.2 & 2.0 & 0.4 & 0.8072 \\
\hline & & & 0.8 & & & 0.7922 \\
\hline & & & 1.6 & & & 0.7199 \\
\hline \multirow[t]{3}{*}{0.5} & 0.1 & 2.0 & 0.3 & 1.0 & 0.4 & 0.5791 \\
\hline & & & & 1.6 & & 0.7388 \\
\hline & & & & 2.2 & & 0.8442 \\
\hline \multirow[t]{3}{*}{0.5} & 0.1 & 2.0 & 0.3 & 2.0 & 0.1 & 0.8809 \\
\hline & & & & & 0.6 & 0.7762 \\
\hline & & & & & 1.2 & 0.6899 \\
\hline
\end{tabular}


Table 3: Outcomes of local Sherwood number $-\phi^{\prime}(0)$ for various parameters

\begin{tabular}{|c|c|c|c|c|c|c|}
\hline$M$ & $\lambda$ & $P r$ & $N t$ & $N b$ & Le & $-\phi^{\prime}(0)$ \\
\hline 0.1 & 0.1 & 2.0 & 0.3 & 0.2 & 2.0 & 1.2414 \\
\hline 0.6 & & & & & & 1.2149 \\
\hline 1.2 & & & & & & 1.1869 \\
\hline \multirow[t]{3}{*}{0.5} & 0.2 & 2.0 & 0.3 & 0.2 & 2.0 & 1.2221 \\
\hline & 0.8 & & & & & 1.2342 \\
\hline & 1.6 & & & & & 1.2484 \\
\hline \multirow[t]{3}{*}{0.5} & 0.1 & 3.0 & 0.3 & 0.2 & 2.0 & 1.3630 \\
\hline & & 4.0 & & & & 1.4850 \\
\hline & & 5.0 & & & & 1.5750 \\
\hline \multirow[t]{3}{*}{0.5} & 0.1 & 2.0 & 0.2 & 0.2 & 2.0 & 1.4036 \\
\hline & & & 0.8 & & & 1.5843 \\
\hline & & & 1.6 & & & 3.0875 \\
\hline \multirow[t]{3}{*}{0.5} & 0.1 & 2.0 & 0.3 & 0.1 & 2.0 & 1.3916 \\
\hline & & & & 0.4 & & 1.5979 \\
\hline & & & & 0.8 & & 1.8989 \\
\hline \multirow[t]{3}{*}{0.5} & 0.1 & 2.0 & 0.3 & 0.2 & 1.4 & 1.1997 \\
\hline & & & & & 1.8 & 1.2468 \\
\hline & & & & & 2.2 & 1.3166 \\
\hline
\end{tabular}

Table 4: Outcomes of local microorganism density number $-\chi^{\prime}(0)$ for various parameters

\begin{tabular}{|c|c|c|c|c|c|c|}
\hline$M$ & $\lambda$ & $\mathrm{Nr}$ & $N c$ & $P e$ & $L b$ & $-\chi^{\prime}(0)$ \\
\hline 0.1 & 0.1 & 0.5 & 0.5 & 0.1 & 2.0 & 1.3662 \\
\hline 0.6 & & & & & & 1.3281 \\
\hline 1.2 & & & & & & 1.2882 \\
\hline \multirow[t]{3}{*}{0.5} & 0.1 & 0.5 & 0.2 & 0.1 & 2.0 & 1.3812 \\
\hline & & & 0.8 & & & 1.3509 \\
\hline & & & 1.6 & & & 1.3387 \\
\hline \multirow[t]{3}{*}{0.5} & 0.1 & 0.5 & 0.5 & 0.4 & 2.0 & 1.4435 \\
\hline & & & & 0.8 & & 2.1046 \\
\hline & & & & 1.6 & & 3.0148 \\
\hline \multirow[t]{3}{*}{0.5} & 0.1 & 0.5 & 0.5 & 0.1 & 1.0 & 0.9147 \\
\hline & & & & & 1.6 & 1.1817 \\
\hline & & & & & 2.2 & 1.4067 \\
\hline \multirow[t]{3}{*}{0.5} & 0.2 & 0.5 & 0.5 & 0.1 & 2.0 & 1.3396 \\
\hline & 0.8 & & & & & 1.3638 \\
\hline & 1.6 & & & & & 1.3924 \\
\hline \multirow[t]{3}{*}{0.5} & 0.1 & 0.2 & 0.5 & 0.1 & 2.0 & 1.3658 \\
\hline & & 0.8 & & & & 1.3368 \\
\hline & & 1.6 & & & & 1.2976 \\
\hline
\end{tabular}




\section{Concluding Remarks}

In this paper, we focus on the radiative Oldroyd-B nanofluid over stretchable disk with swimming microorganisms. The behavior of variable thermal and solutal diffusivity is investigated. Salient features of this work are:

- The larger buoyancy ratio parameter reduces the flow of fluid.

- The velocity field is improved by increasing the velocity ratio parameter and mixed convection parameter.

- Larger Deborah number of retardation time enhances the momentum distribution, while it decreases bioconvection Rayleigh number.

- The heat transformation is improved by dissolving nanomaterials in base fluid [48].

- The increment in the temperature ratio parameter boosts the temperature field.

- The concentration of nanoparticles is boosted with increasing thermophoretic parameters and Arrhenius activation energy.

- The concentration of nanoparticles is a decreasing function of the mixed convection parameter and the Lewis number.

- The larger Peclet number and bioconvection Lewis number decline the microorganism field.

Authors' Contribution: All authors have an equivalent contribution.

Funding Statement: This work is supported by the National Natural Science Foundation of China (Nos. 51876170, 12072257), the Natural Science Basic Research Plan in Shaanxi Province of China (No. 2019JM-343) and the National Key Project (GJXM92579).

Conflicts of Interest: The authors declare that they have no conflicts of interest to report regarding the present study.

\section{References}

1. Choi, S. (1995). Enhancing thermal conductivity of fluids with nanoparticle, ASME Fluids Engineering Division, 231, 99-105.

2. Buongiorno, J. (2006). Convective transport in nanofluids. Journal of Heat Transfer, 128, 240-250. DOI 10.1115/1.2150834.

3. Aminian, E., Moghadasi, H., Saffari, H. (2020). Magnetic field effects on forced convection flow of a hybrid nanofluid in a cylinder filled with porous media: A numerical study. Journal of Thermal Analysis and Calorimetry, vol. 141, 2019-2031. DOI 10.1007/s10973-020-09257-y.

4. Saha, S. K. (2020). Magnetohydrodynamic buoyancy driven $\mathrm{Al}_{2} \mathrm{O}_{3}$-water nanofluid flow in a differentially heated trapezoidal enclosure with a cylindrical barrier. International Communications in Heat and Mass Transfer, 114, 104593. DOI 10.1016/j.icheatmasstransfer.2020.104593.

5. Bhandari, A., Mishra, P. K. (2020). Heat and radiation absorption effects on casson nanofluid flow over a stretching cylinder in the presence of chemical reaction through mathematical modeling. Special Topics \& Reviews in Porous Media: An International Journal, 11(2), 177-188. DOI 10.1615/SpecialTopicsRevPorousMedia.2020029260.

6. Mondal, H., Das, S., Kundu, P. K. (2020). Influence of an inclined stretching cylinder over MHD mixed convective nanofluid flow due to chemical reaction and viscous dissipation. Heat Transfer, 49(4), 21832193. DOI 10.1002/htj.21714. 
7. Basha, H. T., Sivaraj, R., Prasad, V. R., Beg, O. A. (2020). Entropy generation of tangent hyperbolic nanofluid flow over a circular cylinder in the presence of nonlinear boussinesq approximation: A non-similar solution. Journal of Thermal Analysis and Calorimetry, 143(3), 2273-2289. DOI 10.1007/s10973-020-09981-5.

8. Sheikholeslami, M., Arabkoohsar, A., Jafaryar, M. (2020). Impact of a helical-twisting device on the thermal-hydraulic performance of a nanofluid flow through a tube. Journal of Thermal Analysis and Calorimetry, 139(5), 3317-3329. DOI 10.1007/s10973-019-08683-x.

9. Sudarsana Reddy, P., Sreedevi, P. (2020). Impact of chemical reaction and double stratification on heat and mass transfer characteristics of nanofluid flow over porous stretching sheet with thermal radiation. International Journal of Ambient Energy, 1-11. DOI 10.1080/01430750.2020.1712240.

10. Mishra, A., Kumar, M. (2020). Velocity and thermal slip effects on MHD nanofluid flow past a stretching cylinder with viscous dissipation and joule heating. SN Applied Sciences, 2(8), 1-13. DOI 10.1007/s42452-020-3156-7.

11. Khashi'ie, N. S., Waini, I., Zainal, N. A., Hamzah, K., Mohd Kasim, A. R. (2020). Hybrid nanofluid flow past a shrinking cylinder with prescribed surface heat flux. Symmetry, 12(9), 1493. DOI $10.3390 /$ sym12091493.

12. Saeed, A., Tassaddiq, A., Khan, A., Jawad, M., Deebani, W. et al. (2020). Darcy-forchheimer MHD hybrid nanofluid flow and heat transfer analysis over a porous stretching cylinder. Coatings, 10(4), 391. DOI 10.3390/coatings 10040391.

13. Jabbar, M. Y., Hamzah, H. K., Ali, F. H., Ahmed, S. Y., Ismael, M. A. (2020). Thermal analysis of nanofluid saturated in inclined porous cavity cooled by rotating active cylinder subjected to convective condition. Journal of Thermal Analysis and Calorimetry, 144, 1-25. DOI 10.1007/s10973-020-09668-x.

14. Goudarzi, S., Shekaramiz, M., Omidvar, A., Golab, E., Karimipour, A. et al. (2020). Nanoparticles migration due to thermophoresis and brownian motion and its impact on $\mathrm{Ag}-\mathrm{MgO} / \mathrm{Water}$ hybrid nanofluid natural convection. Powder Technology, 375, 493-503. DOI 10.1016/j.powtec.2020.07.115.

15. Ali, F. H., Hamzah, H. K., Hussein, A. K., Jabbar, M. Y., Talebizadehsardari, P. (2020). MHD mixed convection due to a rotating circular cylinder in a trapezoidal enclosure filled with a nanofluid saturated with a porous media. International Journal of Mechanical Sciences, 181, 105688. DOI 10.1016/j.ijmecsci.2020.105688.

16. Bestman, A. R., (1990). Natural convection boundary layer with suction and mass transfer in a porous medium. Internationa Journal of Energy Research, 14, 389-396. DOI 10.1002/(ISSN)1099-114X.

17. Ramesh, G. K. (2020). Analysis of active and passive control of nanoparticles in viscoelastic nanomaterial inspired by activation energy and chemical reaction. Physica A: Statistical Mechanics and its Applications, 550, 123964. DOI 10.1016/j.physa.2019.123964.

18. Abuzaid, D., Ullah, M. Z. (2020). Numerical study for mixed convection flow of oldroyd-b nanofluid subject to activation energy and binary chemical reaction. Thermal Science, 174-174. DOI 10.2298/TSCI200214174A.

19. Waqas, M., Jabeen, S., Hayat, T., Shehzad, S. A., Alsaedi, A. (2020). Numerical simulation for nonlinear radiated eyring-powell nanofluid considering magnetic dipole and activation energy. International Communications in Heat and Mass Transfer, 112, 104401. DOI 10.1016/j.icheatmasstransfer.2019.104401.

20. Khan, M. I., Afzal, S., Hayat, T., Waqas, M., Alsaedi, A. (2020). Activation energy for the carreau-yasuda nanomaterial flow: Analysis of the entropy generation over a porous medium. Journal of Molecular Liquids, 297, 111905. DOI 10.1016/j.molliq.2019.111905.

21. Oldroyd, J. G. (1950). On the formulation of rheological equations of state. Proceedings of the Royal Society A. Mathematical and Physical Sciences, 200(1063), 523-541. DOI 10.1098/rspa.1950.0035.

22. Fröhlich, A., Sack, R. (1946). Theory of the rheological properties of dispersions. Proceedings of the Royal Society A. Mathematical and Physical Sciences, 185(1003), 415-430. DOI 10.1098/rspa.1946.0028.

23. Ibrahim, W., Gadisa, G. (2020). Finite element solution of nonlinear convective flow of oldroyd-b fluid with cattaneo-christov heat flux model over nonlinear stretching sheet with heat generation or absorption. Propulsion and Power Research, 9(3), 304-315. DOI 10.1016/j.jppr.2020.07.001. 
24. Irfan, M., Khan, M., Khan, W. A., Alghamdi, M., Ullah, M. Z. (2020). Influence of thermalsolutal stratifications and thermal aspects of non-linear radiation in stagnation point oldroydb nanofluid flow. International Communications in Heat and Mass Transfer, 116, 104636. DOI 10.1016/j.icheatmasstransfer.2020.104636.

25. Anwar, T., Kumam, P., Khan, I., Thounthong, P. (2020). Heat transfer analysis of unsteady natural convection flow of oldroyd-b model in the presence of newtonian heating and radiation heat flux. IEEE Access, 8, 92479-92489. DOI 10.1109/ACCESS.2020.2989348.

26. Qi, H., Fatima, N., Waqas, H., Saeed, J. (2017). Analytical solution for the flow of a generalized Oldroyd-B fluid in a circular cylinder. Open Journal for Mathematics and Sciences, 1(1), 85-96. DOI 10.30538/oms2017.0009.

27. Waqas, H., Imran, M., Muhammad, T., Sait, S. M., Ellahi, R. (2021). Numerical investigation on bioconvection flow of Oldroyd-B nanofluid with nonlinear thermal radiation and motile microorganisms over rotating disk. Journal of Thermal Analysis \& Calorimetry, 145(2), 523-539. DOI 10.1007/s10973-020-09728-2.

28. Platt, J. R. (1996). Bioconvection patterns in cultures of free-swimming organisms. Science, 133(3466), 1766-1767. DOI 10.1126/science.133.3466.1766.

29. Mondal, S. K., Pal, D. (2020). Gyrotactic mixed bioconvection flow of a nanofluid over a stretching wedge embedded in a porous media in the presence of binary chemical reaction and activation energy. International Journal of Ambient Energy, 1-17. DOI 10.1080/01430750.2020.1814860.

30. Khan, M., Salahuddin, T., Malik, M. Y., Alqarni, M. S., Alqahtani, A. M. (2020). Numerical modeling and analysis of bioconvection on MHD flow due to an upper paraboloid surface of revolution. Physica A: Statistical Mechanics and its Applications, 553, 124231.

31. Ahmad, R., Farooqi, A., Zhang, J. (2020). Analysis of transport and mixing phenomenon to invariant manifolds using LCS and KAM theory approach in unsteady dynamical systems. IEEE Access, 8, 141057141065. DOI 10.1109/Access.6287639.

32. Farooqi, A., Ahmad, R., Khan, A. (2021). A comparative epidemiological stability analysis of predictor corrector type non-standard finite difference scheme for the transmissibility of measles. Results in Physics, 21, 103756. DOI 10.1016/j.rinp.2020.103756.

33. Bary, G., Ahmed, W. (2021). Regio and stereoselective functionalization of alkenes with emphasis on mechanistic insight and sustainability concerns. Journal of Saudi Chemical Society, 25(7), 101260. DOI 10.1016/j.jscs.2021.101260.

34. Ahmad, R., Farooqi, A., Farooqi, R., Khan, I., Sajid, M. et al. (2021). An analytical approach to study the blood flow over a nonlinear tapering stenosed artery in flow of carreau fluid model. Complexity, 21, 1-11.

35. Ahmad, R., Farooqi, A., Zhang, J. (2019). Steady flow of a power law fluid through a tapered non-symmetric stenotic tube. Applied Mathematics and Nonlinear Sciences, 4(1), 249-260. DOI 10.2478/AMNS.2019.1.00022.

36. Sajid, M., Dehua, L., Zhao, X. (2021). Catalytic conversion of xylose to furfural by p-toluenesulfonic acid (pTSA) and chlorides: Process optimization and kinetic modeling. Molecules, 26(8), 2208. DOI 10.3390/molecules26082208.

37. Sajid, M., Dehua, L., Zhao, X. (2020). Organic acid catalyzed production of platform chemical 5hydroxymethylfurfural from fructose: Process comparison and evaluation based on kinetic modeling. Arabian Journal of Chemistry, 13(10), 7430-7444. DOI 10.1016/j.arabjc.2020.08.019.

38. Song, Y. Q., Khan, S. A., Imran, M., Waqas, H., Khan, S. U. et al. (2021). Applications of modified darcy law and nonlinear thermal radiation in bioconvection flow of micropolar nanofluid over an off centered rotating disk. Alexandria Engineering Journal, 60(5), 4607-4618. DOI 10.1016/j.aej.2021.03.053.

39. Farooq, U., Waqas, H., Khan, M. I., Khan, S. U., Chu, Y. M. et al. (2021). Thermally radioactive bioconvection flow of carreau nanofluid with modified cattaneo-christov expressions and exponential space-based heat source. Alexandria Engineering Journal, 60(3), 3073-3086. DOI 10.1016/j.aej.2021.01.050.

40. Tlili, I., Waqas, H., Almaneea, A., Khan, S. U., Imran, M. (2019). Activation energy and second order slip in bioconvection of Oldroyd-B nanofluid over a stretching cylinder: A proposed mathematical model. Processes, 7(12), 914. DOI 10.3390/pr7120914. 
41. Waqas, H., Khan, S. U., Shehzad, S. A., Imran, M., Tlili, I. (2020). Activation energy and bioconvection aspects in generalized second-grade nanofluid over a Riga plate: A theoretical model. Applied Nanoscience, 10(12), 4445-4458. DOI 10.1007/s13204-020-01332-y.

42. Hafeez, A., Khan, M., Ahmed, A. ( (2020). Stagnation point flow of radiative oldroyd-b nanofluid over a rotating disk. Computer Methods and Programs in Biomedicine, 191, 105342. DOI 10.1016/j.cmpb. 2020.105342.

43. Waqas, H., Imran, M., Muhammad, T., Sait, S. M., Ellahi, R. (2020). Numerical investigation on bioconvection flow of Oldroyd-B nanofluid with nonlinear thermal radiation and motile microorganisms over rotating disk. Journal of Thermal Analysis and Calorimetry, 145(2), 1-17. DOI 10.1007/s10973-020-09728-2.

44. Ahmed, A., Khan, M., Ahmed, J., Hafeez, A., Iqbal, Z. (2020). Unsteady stagnation point flow of Maxwell nanofluid over stretching disk with joule heating. Arabian Journal for Science and Engineering, 45(7), 1-12. DOI 10.1007/s13369-020-04468-9.

45. Zhou, S. S., Bilal, M., Khan, M. A., Muhammad, T. (2021). Numerical analysis of thermal radiative maxwell nanofluid flow over-stretching porous rotating disk. Micromachines, 12(5), 540. DOI 10.3390/mi12050540.

46. Bary, G., Ru, P., Zhang, W. (2019). Normalized multi-pion hanbury-brown-twiss correlation functions of pion emitting sources with bose-einstein condensation. Journal of Physics G: Nuclear and Particle Physics, 46, 115107. DOI 10.1088/1361-6471/ab449a.

47. Khan, S. A., Waqas, H., Naqvi, S., Alghamdi, M., Al-Mdallal, Q. (2021). Cattaneo-christov double diffusions theories with bio-convection in nanofluid flow to enhance the efficiency of nanoparticles diffusion. Case Studies in Thermal Engineering, 26, 101017. DOI 10.1016/j.csite.2021.101017.

48. Maskeen, M. M., Zeeshan, A., Mehmood, O. U., Hassan, M. (2019). Heat transfer enhancement in hydromagnetic alumina-copper/water hybrid nanofluid flow over a stretching cylinder. Journal of Thermal Analysis and Calorimetry, 138(2), 1127-1136. DOI 10.1007/s10973-019-08304-7. 